\title{
US Accelerator R\&D Program Toward Intensity Frontier Machines
}

\author{
Vladimir Shiltsev ${ }^{1}$ \\ Fermi National Accelerator Laboratory \\ PO Box 500, Batavia IL 60510, USA \\ E-mail: shiltsev@fnal.gov
}

The 2014 P5 report [1] indicated the accelerator-based neutrino and rare decay physics research as a centrepiece of the US domestic HEP program. Operation, upgrade and development of the accelerators for the near-term and longer-term particle physics program at the Intensity Frontier face formidable challenges. Here we discuss key elements of the accelerator physics and technology R\&D program toward future multi-MW proton accelerators.

38th International Conference on High Energy Physics

3-10 August 2016

Chicago, USA

\footnotetext{
${ }^{1}$ Fermi Research Alliance, LLC operates Fermilab under contract no. DE-AC0207CH11359 with the U.S. Department of Energy
} 


\section{Introduction}

The 2014, the Particle Physics Project Prioritization Panel (P5) report [1] identified the top priority of the domestic intensity frontier high-energy physics for the next 20-30 years to be a high energy neutrino program to determine the mass hierarchy and measure CP violation, based on the Fermilab accelerator complex which needs to be upgraded for increased proton intensity. To this end, a new beam line - the Long Baseline Neutrino Facility (LBNF) - and new experiment - the Deep Underground Neutrino Experiment (DUNE), located in the Sanford Underground Research Facility (SURF) - are being planned [2]. This will be a truly international collaboration, including contributions from 150 institutions in 27 countries. The P5 physics goals require about $900 \mathrm{kt} \cdot \mathrm{MW}$-years of exposure (product of the neutrino detector mass, average proton beam power on the neutrino target and data taking period) and that can be achieved assuming a 40 kton Liquid Argon detector and accelerator operation with the eventual multi MW beam power. Construction of the PIP-II SRF $800 \mathrm{MeV}$ linac [3] is expected to address the near-term challenges. PIP-II will increase the Booster per pulse intensity by $50 \%$ and allow delivery 1.2 MW of the $120 \mathrm{GeV}$ beam power from the Fermilab's Main Injector, with power approaching 1 MW at energies as low as $60 \mathrm{GeV}$, at the start of DUNE/LBNF operations ca 2023. Extensive accelerator R\&D program towards multi-MW beams has been started in the US and it has three components: demonstration of novel techniques for high-current beam accelerators at IOTA, cost-effective SC RF and high-power targetry (HPT).

\section{Experimental R\&D with high brightness beam at IOTA ring}

Progress of the Intensity Frontier accelerator based HEP is hindered by fundamental beam physics phenomena such as space-charge effects, beam halo formation, particle losses, transverse and longitudinal instabilities, beam loading, inefficiencies of beam injection and extraction, etc. The Integrable Optics Test Accelerator (IOTA) facility at Fermilab [4] is being built as a unique test-bed for transformational R\&D towards the next generation high-intensity proton facilities see Fig. 2. The experimental accelerator R\&D at the $40 \mathrm{~m}$ circumference IOTA ring - see Fig.1 - with high brightness $70 \mathrm{MeV} / \mathrm{c}$ protons and $150 \mathrm{MeV} / \mathrm{c}$ electrons, augmented with corresponding modeling and design efforts has been started at Fermilab in collaboration more than two dozen universities, National and international partners [5].

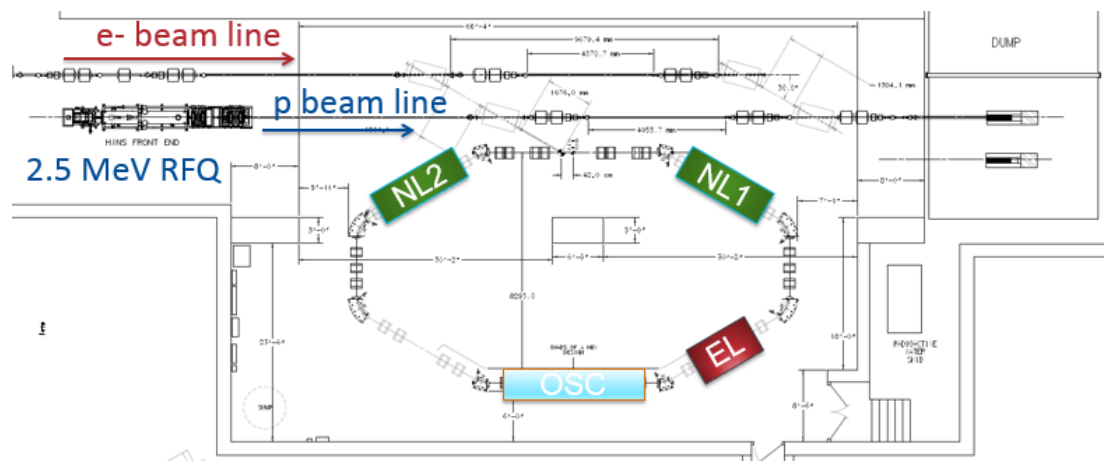

Figure 1: IOTA ring, its electron and proton injection lines and experimental areas.

The goal of the IOTA research program is to carry out experimental studies of transformative techniques to control proton beam instabilities and losses, such as integrable optics [6] with 
non-linear magnets and with electron lenses, and space-charge compensation with electron lenses and electron columns $[7,8]$ at beam intensities and brightness 3-4 times the current operational limits, i.e., at the space-charge parameter $\Delta Q_{S C}$ approaching or even exceeding 1 . Several experiments are planned at IOTA: i) Test of Integrable Optics (IO) with Electrons with a goal to create IO accelerator lattice with several additional integrals of motion (angular momentum and McMillan-type integrals, quadratic in momentum); ii) IO with Non-linear Magnets, Test with Protons will demonstrate nonlinear integrable optics with protons with a large betatron frequency spread $\Delta Q_{s c}>1$ and stable particle motion in a realistic accelerator design; iii) IO with e-lens(es), Tests with Protons to demonstrate IO with non-Laplacian electron lenses with the electron charge distribution as $n(r)=1 /\left(1+r^{2}\right)^{2}$ to obtain a large betatron frequency spread $\Delta Q_{S C}>1$ and stable particle motion in a realistic accelerator design; iv) Space-Charge Compensation (SCC) with e-lens(es), Test with Protons has the main goal of demonstrating SCC with Gaussian ELs with protons with a large betatron frequency spread $\Delta \mathrm{Q}>0.5$ and stable particle motion in a realistic accelerator design. Similar SCC tests are envisioned with electron columns [8].

In 2016, the IOTA team has commissioned $50 \mathrm{MeV}$ SRF electron pre-injector [5]. Operation of the IOTA ring with $150 \mathrm{MeV}$ electrons in planned for 2017, and with protons in 2019.

\section{Cost effective SRF technology}

Superconducting RF is the state-of-the-art technology with an unmatched capability to provide up to $100 \%$ beam duty factor and large apertures to preserve the beam quality. In the past, the SRF R\&D program has been focused on improving the accelerating gradients in "traditional" Nb structures, extending from $3 \mathrm{MV} / \mathrm{m}$ to some $35 \mathrm{MV} / \mathrm{m}$. The demands of the Intensity Frontier accelerators shift of focus towards decreasing the costs of SRF construction and operation through: a) nitrogen doping for ultra-high Q cavities, which opens up more than a factor of two in the quality factors $(\mathrm{Q})$ of bulk niobium cavities and, therefore, postential for savings in cryogenics capital and operational costs [9]; b) development $\mathrm{Nb}_{3} \mathrm{Sn}$ cavities for $4.2 \mathrm{~K}$ operation, following the proof-of-principle demo that Nb3Sn cavities could provide the same quality factors at $>4.2 \mathrm{~K}$ as bulk niobium cavities do at $2 \mathrm{~K}$ [10]; c) using $\mathrm{Nb} / \mathrm{Cu}$ composite material and monolithic techniques of cavity manufacturing; these avenues promise a factor of $>2$ reduction in cavity material and manufacturing costs with performance comparable to bulk $\mathrm{Nb}$ cavities. It was recently shown that $1.3 \mathrm{GHz} \mathrm{Nb}-\mathrm{Cu}$ composite based spun cavities can sustain high accelerating gradients. Fermilab, in collaboration with Cornell University, will use the existing Nb-Cu sheets to spin the cavities at INFN(Italy) or US industry (e.g. AES) to complete the $650 \mathrm{MHz}$ cavities with flanges as the first step followed by scaling to $325 \mathrm{MHz}$ if successful. Recent breakthrough in $\mathrm{Nb}$ film deposition technology allows films of unprecedented quality with the residual resistivity ratio (RRR) approaching or exceeding 200-300, which is currently the standard for bulk SRF cavities. The RF properties of these films will be tested, and after confirmation of low surface resistance of the samples, a prototype $650 \mathrm{MHz} \mathrm{Nb} / \mathrm{Cu}$ cavity will be built and studied.

\section{High power targetry R\&D}

Mega-watt class target facilities present many technical challenges, including: radiation damage, rapid heat removal, high thermal shock response, highly non-linear thermo-mechanical simulation, radiation protection, and remote handling [11]. The major goal of the envisioned R\&D program for the next decade is to enable well-justified design simulations of high intensity beam/matter interactions using realistic, irradiated material properties for the purposes of designing and predicting lifetimes of multi-MW neutrino and muon target components and systems. 
This requires: a) irradiated material properties to be measured/evaluated for relevant targetry materials over a range of temperatures $(300-1300 \mathrm{~K})$, radiation damage $(0.1-20$ DPA (Displacements Per Atom)) and relevant helium production rates (500 - 5000 atomic parts per million/DPA); b) thermal shock response to be evaluated for relevant targetry materials over a range of strain rates $\left(100-10000 \mathrm{~s}^{-1}\right)$; c) development and validation of simulation techniques to model material response to beam over the time of exposure (accounting for accumulation of radiation damage and high spatial gradients); d) development of enabling technologies in target materials, manufacturing techniques, cooling technologies, instrumentation, radiation protection, and related systems to meet the targetry challenges of multi-MW and/or high intensity ( $>500 \mathrm{MW} / \mathrm{m}^{3}$ peak energy deposition) requirements of future target facilities.

Radiation damage studies include investigations of materials of high interest (currently graphite, beryllium, tungsten and titanium alloys) under the RaDIATE R\&D program [12]. The most major of these activities involve Post-Irradiation Examination (PIE) of previously irradiated materials recovered from spent target components (e.g. NuMI proton beam window), low-energy ion and high energy proton irradiations at available beam facilities (e.g. Brookhaven Linac Isotope Producer [13]), and experiments designed to help correlate low energy ion irradiations to high energy proton irradiations.

Thermal shock response studies include in-beam thermal shock experiments of various grades of commercially pure beryllium at the HiRadMat Facility [14] at CERN (e.g. HRMT-24, "BeGrid" [15]) and high strain rate testing of candidate materials to develop strength and damage models.

\section{References}

1. Building for Discovery (P5 Report, May 2014); see at http://science.energy.gov/hep/hepap/reports/

2. LBNF and DUNE Conceptual Design Report, arXiv:1601.05471

3. The PIP-II Reference Design Report (2015) https://indico.fnal.gov/getFile.py/access?resId=0\&materialId=2\&confId=9939

4. M.Church, et al (eds.), ASTA Facility Proposal, Fermilab-TM-2568 (2013)

5. http://fast.fnal.gov/

6. V.Danilov, S.Nagaitsev, Nonlinear accelerator lattices with one and two analytic invariants, Physical Review Special Topics-Accelerators and Beams 13(8) (2010) 084002

7. V.Shiltsev, Electron Lenses for Super-Colliders (New York: Springer, 2016)

8. V.Shiltsev, et al, The use of ionization electron columns for space-charge compensation in high intensity proton accelerators. AIP Conf. Proc. 1086 (2009) 649

9. A.Grassellino A, et al, Nitrogen and argon doping of niobium for superconducting radio frequency cavities: a pathway to highly efficient accelerating structures, Supercond. Sci. Technol., 26 (2013) 102001 (Rapid Communication)

10. S. S. Posen, et al, Proof-of-principle demonstration of Nb3Sn superconducting radiofrequenc cavities for high Q0 applications, Applied Physics Letters 106 (2015) 082601

11. P.Hurh, et al, Targetry Challenges at Megawatt Proton Accelerator Facilities, Proc. IPAC2013 (Shanqhai, China, 2013) 3484

12. http://radiate.fnal.gov/

13. N.Simos, et al, Studies of Material Properties Under Irradiation at BNL BLIP, arxiv:1202.3799

14. A. Fabich et al., First Year of Operations in the HiRadMat Irradiation Facility at CERN, Proc. of IPAC'13 (Shanghai, China, 2013) 3415

15. K. Ammigan et al., Examination of Beryllium under Intense High Energy Proton Beam at CERN's HiRadMat Facility, Proc. of IPAC'15 (Richmond, VA, USA, 2015) 3289 